\title{
Stemming the losses from COVID-19
}

\author{
The SARS-CoV-2 pandemic has posed one of the greatest scientific challenges to our society in a century. \\ The accompanying disruption will have a disproportionally large effect on the careers of early-stage investigators \\ and will require concrete action to preserve a generation of scientists.
}

\begin{abstract}
$\mathrm{n}$ this issue of Nature Metabolism, we begin our three-part series profiling early-career investigators (L. Finley and

L. Kazak, Nat. Metab. https://doi. org/10.1038/s42255-020-0221-x; 2020). Their career journeys, like those of all other scientists, have been interrupted by the SARS-CoV-2 outbreak. How can we begin to tally the costs of a generational event such as this? Months of restricted laboratory access, travel bans and cancelled conferences, as well as the emotional toll of quarantine on scientists, will have unimaginable ramifications for our scientific community for years to come.

These years will be marked by loss. The lives that we have lost to COVID-19 in our scientific, medical and global communities have already been tremendous. Yet, an unspoken cost of the ensuing chaos lies in what is lost for researchers of all stages: missing data from submitted manuscripts and grant proposals; missing students and trainees as a result of travel restrictions; and missing funding because of a looming global economic downturn.
\end{abstract}

Early-career investigators, however, are a particularly vulnerable group. They, along with senior postdocs, are under tremendous pressure to produce data and papers, to network and establish name recognition, and to secure funding. Our current funding and institutional infrastructures lack the safeguards for early-stage investigators and senior postdocs necessary to account for force majeure, such as a plague. Although established labs may weather fluctuations in funding, personnel and publications, new labs have always had to make do with less, and many will undoubtedly lack the resources and flexibility to endure a disruption on this scale. Moreover, groups that historically lack access to resources, particularly those led by investigators from gender, sexual and racial minorities, risk losing hard-fought gains in parity and representation, the effects of which we are already beginning to ascertain from emerging data (S. E. Acton, A. J. D. Bell, C. P. Toseland and A. Twelvetrees, eLife $\mathbf{8}$, e46827; 2019 and https://www.natureindex. com/news-blog/decline-women-scientistresearch-publishing-production-coronaviruspandemic).

If we can glean anything from the narratives of the six featured early-stage investigators, it is that, although tight budgets, looming deadlines and bare-bones personnel are nothing new to research, a pandemic brings a new set of challenges that will require a new set of solutions.

Publications are a cornerstone of scientific career progression, and we, along with many other journals, have waived deadlines, extended revision periods and taken a pragmatic approach to our editorial instructions to account for inevitable disruptions in manuscript preparation, experiments and data collection. As global economies sputter, the budgets of universities and funding agencies will become pinched. Fellowships for postdocs and funding packages for early-career investigators should be extended to account for this 'lost year.' Long-term departmental recruiting targets cannot be abandoned, and current and near-future vacancies should continue to be filled and funded. Tenure consideration periods also must reflect potential disruptions in lab productivity and be extended.

This effort will require a coordinated and multifactorial approach, and although some solutions, such as extending deadlines or promotion periods, will have few budgetary effects, the most impactful of these solutions will require a reassessment of fund allocation for departments at a time when shortfalls are expected. The fiscal inroads painstakingly made by the scientific community over the past 30 years are at risk. To cede ground at a time when scientific research is predicting, discovering, modelling and providing concrete clinical solutions for the COVID-19 pandemic would leave our global society ill prepared for future crises that require the full mobilization of our collective scientific resources.

In times such as these, when the best minds are needed to work towards solving the coming problems, we cannot allow this global disruption to squeeze a generation of scientists out of academia. Scientists in all stages of their careers bring more than knowledge and expertise-they bring invaluable insight that has been uniquely moulded by personal circumstances, which allows them to approach problems differently and see novel solutions.

This may not be the last pandemic of our lifetimes, but we will need the next generation of our best and brightest at the helm to guide us through the unknown challenges of the future.

Published online: 19 June 2020

https://doi.org/10.1038/s42255-020-0235-4 